\title{
Ruptured mucocele of appendix
}

\begin{abstract}
Although uncommon, primary appendiceal neoplasms often result in clinical symptoms that may lead to abdominal imaging. Acute appendicitis from luminal obstruction is the most common manifestation for most tumor types. Sometimes, patient with Mucocele can present with confusing symptoms, manifestations include intussusception, a palpable mass, gastrointestinal bleeding, increasing abdominal girth (from pseudomyxoma peritonei), and secondary genitourinary complications. Asymptomatic appendiceal neoplasms may be discovered incidentally. Mucoceles from either benign or malignant mucinous neoplasm [1-2] represent the majority of appendiceal tumors detected at imaging but are the least likely to manifest as appendicitis. Pseudomyxoma peritonei is a common manifestation of mucinous adenocarcinoma.
\end{abstract}

Keywords: appendix, computed tomography, mucocele, sonography, magnetic resonance imaging
Volume 2 Issue 4 - 2015

\section{Sushila Ladumor, Adham Darwish,Ala \\ Kamble, Maysa Abdul Hafeez \\ Hamad Medical Corporation Clinical Imaging, Qatar}

Correspondence: Sushila Ladumor, Dr. Sushila Hamad Medical Corporation Clinical Imaging, HGH P.O. Box 3050 Doha, Qatar, Tel 9746430597,Email drsbladumor@yahoo.com

Received: February 27, 2015 | Published: April 08, 2015
Abbreviations: USG, ultra sonography; CT, computed tomography; MRI, magnetic resonance imaging; AM, appendiceal mucocele; AA, acute appendicitis; $\mathrm{R} / \mathrm{O}$, rule out; RIF, right iliac fossa; $\mathrm{D} / \mathrm{D}$, differential diagnosis

\section{Introduction}

Appendiceal mucocele (AM) is a rare entity that can present with a variety of clinical symptoms or occur as an incidental surgical finding. $\mathrm{AM}$ is a progressive dilatation of the appendix from the intraluminal accumulation of the mucoid substance. Preoperative diagnosis that distinguishes AM from acute appendicitis (AA) is essential for the best choice of surgical approach (open vs laparoscopic) to prevent peritoneal dissemination and perform the appropriate surgery.

\section{Case presentation}

$51 \mathrm{yr}$ old male patient presented in Emergency Department with right iliac fossa pain and tenderness with fever.

\section{Local examination}

Right Iliac Fossa tenderness, Vital signs were within normal limit apart from rising temperature of about $39^{\circ} \mathrm{C}$.

\section{Biochemistry profile}

Elevated white blood cell count, other laboratory tests, including CEA, CA 19-9 and CA 125 were unremarkable.

Routine investigation

X-Ray chest: No abnormality detected.

\section{Description and diagnosis}

Patient referred to USG to R/O Appendicitis and US revealed echogenic mass with fluid collection RIF D/D includes ruptured appendix with appendicitis /abscess and other possibility of Mucocele of appendix with rupture in view of dilated appendiceal lumen of about $3.6 \mathrm{~cm}$.

\section{Patient referred to CT abdomen after USG for further evaluation}

CT post-contrast axial and coronal reconstructed images reveal dilated appendix with lumen measures about $3.6 \mathrm{~cm}$ (white arrow in a) and demonstrates high density fluid with density measures about 26HU with peripheral rim of high density (black arrow in b) represents calcification. There was focal luminal defect with localized collection and fat stranding noted. No abnormal focal enahancement. Findings are in favor of ruptured Mucocele of appendix with adjacent collection and possible underlying appendicitis. MRI was requested for confirmation and rule out occult pathology (Figure 1).

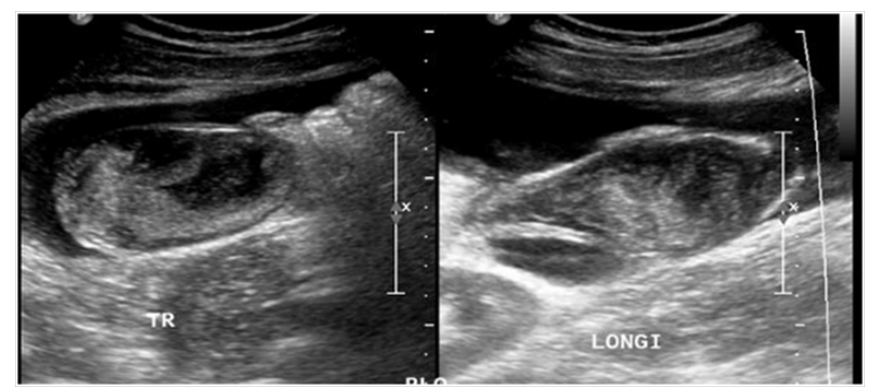

Figure I CT Scan of abdomen after USG Axial \& coronal images.

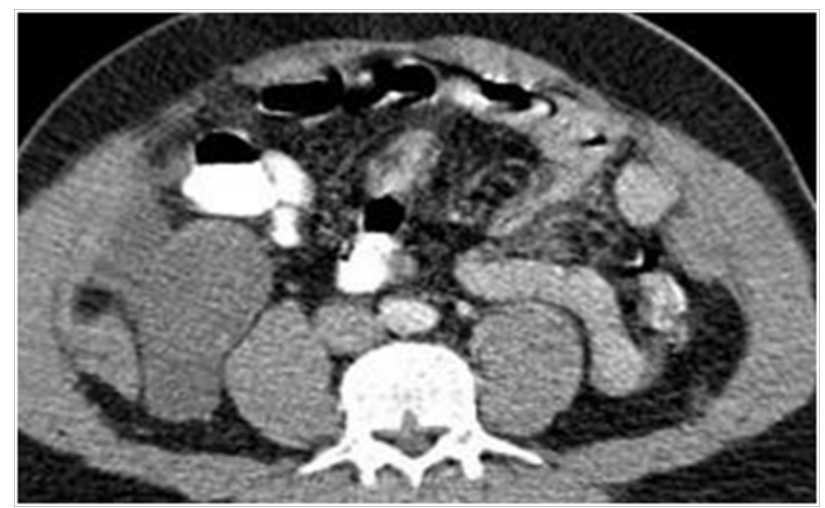

Figure IA MRI-T2 weighted image.

MRI reveal dialted tubular structure in right iliac fossa communicating with the cecum shows high signal with periphreal hypointense rim in T2 weighted images (a,b\&c) keeping with known calcification seen by CT. Post-contrast axial image (b) shows periphral enhancement. Focal defect in wall (shown by arrow in c) which is continuous with surrounding fluid confirm rupture of mucocele (Figure 2). 


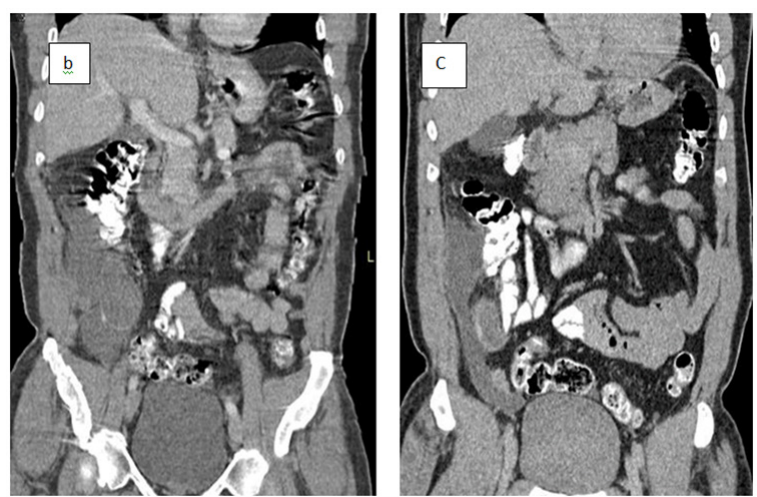

Figure IB \& IC CT scan images of abdomen.
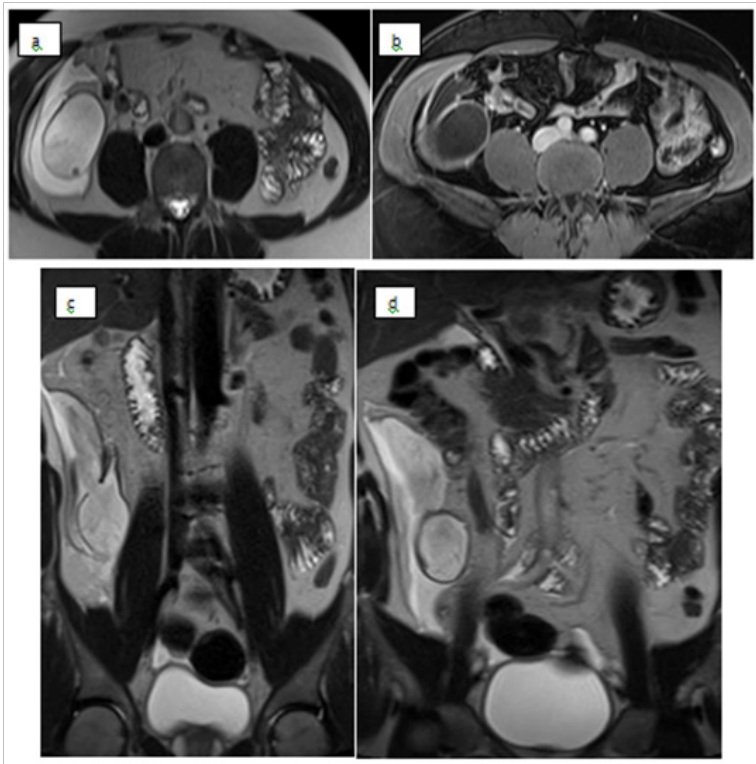

Figure $2 \mathrm{MRI}$ images.

\section{Differential diagnosis}

The other differential diagnosis includes enteric duplication cyst, mesenteric cyst, abscess, hematoma and Lymphocele. In female patient D/D also includes ovarian cyst and Hydrosalpinx. Enteric duplication cyst, mesenteric cyst and lymphocele usually show clear fluid and with no calcification in wall. Enteric duplication cyst usually present in earlier age and mesenteric cyst has septations which are better seen by US and MRI than CT. Lymphocele usually occur postoperatively, particularly in post-kidney transplant in RIF. No history of trauma or other bleeding disorder to suggest hematoma. Possibility of abscess was kept by USG, however in CT and MRI findings of ruptured Mucocele of appendix was confirmed by typical findings.

\section{Treatment}

"Patient underwent appendicaectomy and histopathology of the resected sample revealed low grade mucinous neoplasm with low risk of recurrence". Patient came after 5month with history of left renal colic, so non enhanced CT demonstrae tiny left ureteric stone, otherwise within normal limit. Patient again came back with abdominal pain after 10 month so CT was requested as patient had history of ruptured mucocele with spilling into paritoneal cavity which has high probability that pseudomyxoma peritonei may develop and it reveal development of Pseudomyxoma peritonei with presence of minimal ascites and scalloping of liver and spleen. Patient lost followup after last CT.

\section{Discussion}

Appendiceal mucocoeles occur when there is abnormal accumulation of mucous within the appendix. The tenacious and viscous mucous causes obstruction of the appendiceal neck and results in dilatation of the lumen. The term mucocoele refers to cystic dilatation of the appendix filled with mucin resulting from proximal obstruction of the appendix lumen. Appendiceal mucoceles are rare and are characterized by a distended, mucus-filled appendix. The course and prognosis of appendiceal mucoceles relate to their histologic subtypes. An appendiceal mucocele refers to any lesion that is characterized by a distended, mucus-filled appendix.

Appendiceal mucocele is a rare disease. Sometimes it is discovered accidentally and sometimes it resembles acute appendicitis. Correct diagnosis before surgery is very important for the selection of adequate surgical treatment to avoid intraoperative and postoperative complications. Ultrasonography, and particularly computed tomography, should be used extensively for this purpose. If mucocele is treated incorrectly pseudomyxoma peritonei, which is characterized by malignant process, may develop. Mucoceles may be due to nonneoplastic or neoplastic lesions. ${ }^{1,2}$ The incidence is $0.2 \%$ to $0.7 \%$ of all appendectomied specimens. ${ }^{3}$ There are 4 histologic types of appendiceal mucocele: retention cyst, mucosal hyperplasia, mucinous cystadenoma, and mucinous cystadenocarcinoma.

This disease does not have a typical clinical picture. Sometimes the patient has pain in the lower right quadrant of the abdomen, therefore a surgeon may mistaken it for acute appendicitis. This is one of the most common surgical diseases. It is important to differentiate between these two pathologies before surgery and select adequate surgical tactics. If treated improperly, the mucocele may progress, epithelial cells may escape into the peritoneal cavity, and pseudoyxoma peritonei may develop, which has a high mortality. The clinical flow of the disease does not have a specific picture. It often flows asymptomatically. In about $50 \%$ of cases it is discovered accidentally during radiologic and endoscopic examinations or at surgery. A patient's clinical symptoms may include pain in the right lower quadrant of the abdomen, palpable abdominal mass, nausea, vomiting, weight loss, gastrointestinal bleeding, and signs of intussusception of the intestines. ${ }^{2-8}$

Preoperative diagnosis of appendicular mucocele is very important for the selection of an adequate surgical method to prevent peritoneal dissemination, to prevent intraoperative and postoperative complication, and repeated surgery. USG, computed tomography (CT), and colonoscopy is used for diagnostics. USG is the first-line diagnostic method for patients with acute abdominal pain USG can be used to differentiate between mucocele and acute appendicitis. One of the cardinal principles of surgical treatment of this disease is that intact mucoceles do not pose a threat for the patient. If it is perforated in the peritoneal cavity, there is a high probability that pseudomyxoma peritonei will develop, for which treatment is very problematic and long-term.

\section{Conclusion}

In conclusion, appendiceal mucocele is a rare disease and has a clinical picture that resembles acute appendicitis. A correct diagnosis before surgery is very important for the selection of surgical technique 
to avoid severe intraoperative and postoperative complications. USG, particularly CT, should be used extensively for this purpose. In a female patient with right adnexal cystic lesion dedicated search must be made for appendix and base of cecum.

\section{Acknowledgments}

None.

\section{Conflicts of interest}

Authors declare that there is no conflict of interest.

\section{References}

1. Louis-Michel Wong Kee Song, Norman EM. Appendiceal mucoceles. UpToDate; 2014.

2. Demetrashvili Z, Chkhaidze M, Khutsishvili K, et al. Mucocele of the appendix: case report and review of literature. Int Surg. 2012;97(3):266-269.
3. Rangarajan M, Palanivelu C, Kavalakat AJ, et al. Laparoscopic appendectomy for mucocele of the appendix. Report 8 cases. Indian $J$ Gastroenterol. 2006;25(5):256-257.

4. Pitiakoudis M, Tsaroucha AK, Mimidis K, et al. Mucocele of the appendix: a report of five cases. Tech Coloproctol. 2004;8(2):109-112.

5. Zanati SA, Martin JA, Baker JP, et al. Colonoscopic diagnosis of mucocele of the appendix. Gastrointest Endosc. 2005;62(3):452-456.

6. Madwed D, Mindelzun R, Jeffrey RB. Mucocele of the appendix: Imaging findings. Am J Roentgenol.1992;59(1):69-72.

7. Dachman AH, Lichtenstein JE, Friedman AC. Mucocele of the appendix and pseudomyxoma peritonei. Am J Roentgenol. 1985;144(5):923-929.

8. Skaane P, Ruud TE, Haffner J. Ultrasonographic features of mucocele of the appendix. J Clin Ultrasound. 1988;16(8):584-587. 